International Mathematical Forum, Vol. 9, 2014, no. 13, 631 - 638

http://dx.doi.org/10.12988/

http://dx.doi.org/10.12988/imf.2014.310186

\title{
L-Fuzzy Multivalued Mapping
}

\author{
M. A. Hebeshi \\ Mathematics Department, Faculty of science \\ Sohag university, Egypt
}

Copyright (c) 2014 M. A. Hebeshi. This is an open access article distributed under the Creative Commons Attribution License, which permits unrestricted use, distribution, and reproduction in any medium, provided the original work is properly cited.

\begin{abstract}
In this paper, we introduce a new definition of $L$-fuzzy multivalued mapping and investigate some of their properties.
\end{abstract}

Mathematics Subject Classification: 54A40, 54C08, 54C60

Keywords: $L$-Fuzzy sets, $L$-fuzzy multivalued mapping

\section{Introduction}

In 1988, Ottoy and Kerre introduced the concept of a fuzzy multivalued mapping [2], based on the observation that every fuzzy relation from a universe $X$ into a universe $Y$ can be characterized by means of a unique mapping from $X$ into $I^{Y}$ (with $I=[0,1]$ ) and vice versa. Due to this identification, the whole machinery of Berge's multivalued mappings can be extended to fuzzy multivalued mappings. In [2], Ottoy and Kerre also defined the direct images, the lower and upper inverse images of fuzzy sets under a fuzzy multivalued mapping, and listed some elementary properties. Recently, we have taken up this study again, and have added some additional properties to this list, mainly concerning the direct and the lower inverse images [4]. Such a repository of properties and relationships comes in very handly, as we have experienced in our sucessful extension of Berge's pure and stable sets with respect to multivalued mappings to fuzzy multivalued mappings $[1,3]$. The purpose of this paper is to share this arsenal of properties with other recearchers. We will therefore discuss them in detail and provide, for the first time, proofs where required. 


\section{Preliminaries}

Throughout this paper, let $X$ be a nonempty set and $L=(L, \leq, \vee, \wedge, \perp, \top)$ a completely distributive lattice where $\perp$ (resp. $\top$ ) denotes the universal lower (resp. upper ) bound.

Definition 2.1 [6]CQML, the category of complete quasi-monoidal lattices, comprises the following data, where composition and identities are taken from SET:

(1) Objects: $(L, \leq, \odot)$ where $\odot: L \times L \rightarrow L$ is isotone and $\top \odot \top=\top$.

(2) Morphisms: All SET morphisms preserves $\odot, \top$ and arbitrary $\vee$.

Definition 2.2 [6] Categories related to CQML.

(1) QUML, the category of quasi-uniform monoidal lattices is the full subcategory of CQML for which $\odot$ is associative, commutative and $T$ is identity.

(2) DQML, the category of deMorgan quasi-monoidal lattices is the full subcategory of CQML for which $*$ is an order-reversing involution and each morphism preserves the involution.

(3) QUANT, the category of quantales is the full subcategory of CQML for which $\odot$ is distributive over arbitrary joins, i.e.,

$$
\left(\bigvee_{i \in \Gamma} r_{i}\right) \odot s=\bigvee_{i \in \Gamma}\left(r_{i} \odot s\right)
$$

(4) QUANT, the category of coquantales is the full subcategory of CQML for which $\odot$ is distributive over arbitrary meets, i.e.,

$$
\left(\bigwedge_{i \in \Gamma} r_{i}\right) \odot s=\bigwedge_{i \in \Gamma}\left(r_{i} \odot s\right) .
$$

(5) DQUAT, the category of deMorgan, quasi-uniform monoidal quantales. In this paper, for each $(L, \leq, \odot, *) \in$ DQUAT, we define $x \oplus y=$ $\left(x^{*} \odot y^{*}\right)^{*}$.

(6) DBIQUAT =DQUAT $\cap$ COQUANT.

(7) CMVAL, the category of complete MV-algebra is the full subcategory of DBIQUAT for which it satisfies

$(\mathrm{MV})(x \mapsto y) \mapsto y=x \vee y$, for all $x, y \in L$ where $x \mapsto y$ is defined by $x \mapsto y=\bigvee\{z \mid x \odot z \leq y\}$ and $x^{*}=x \mapsto \perp$.

Definition $2.3[5]$ Let $(L, \leq, \odot, \oplus, *) \in \mid$ DQUAT $\mid$ and $\phi: X \rightarrow Y$ be a function. For each $x, y, z \in L,\left\{y_{i} \mid i \in \Gamma\right\} \subset L, f, g, \in L^{X}$ and $f_{i} \in L^{Y}$ we have:

(1) If $y \leq z,(x \odot y) \leq(x \odot z)$ and $(x \oplus y) \leq(x \oplus z)$. 
(2) $x \odot y \leq x \wedge y \leq x \vee y \leq x \oplus y$.

(3) $\bigwedge_{i \in \Gamma} y_{i}^{*}=\left(\bigvee_{i \in \Gamma} y_{i}\right)^{*}$ and $\bigvee_{i \in \Gamma} y_{i}^{*}=\left(\bigwedge_{i \in \Gamma} y_{i}\right)^{*}$

(4) $x \oplus\left(\bigwedge_{i \in \Gamma} y_{i}\right)=\bigwedge_{i \in \Gamma}\left(x \oplus y_{i}\right)$.

(5) $\phi^{\rightarrow}(f \odot g) \leq \phi^{\rightarrow}(f) \odot \phi^{\rightarrow}(g)$ with equality if $\phi$ is injective.

(6) $\phi^{\leftarrow}\left(\odot_{i \in \Gamma} f_{i}\right)=\odot_{i \in \Gamma} \phi^{\leftarrow}\left(f_{i}\right)$ and $\phi^{\leftarrow}\left(\oplus_{i \in \Gamma} f_{i}\right)=\oplus_{i \in \Gamma} \phi^{\leftarrow}\left(f_{i}\right)$, $\Gamma$ is finite.

(7) For $(L, \leq, \odot, \oplus, *) \in \mid$ DBIQUAT $\mid$, we have $x \oplus\left(\bigvee_{i \in \Gamma} y_{i}\right)=\bigvee_{i \in \Gamma}\left(x \oplus y_{i}\right)$.

(8) For $(L, \leq, \odot, \oplus, *) \in \mid$ DBIQUAT $\mid$, we have $\phi \rightarrow(f \oplus g) \leq \phi^{\rightarrow}(f) \oplus \phi^{\rightarrow}(g)$ with equality if $\phi$ is injective.

\section{$3 \quad L$-fuzzy multivalued mapping}

Definition 3.1 An $L$-fuzzy multivalued mapping ( $L$-FMM, for short) $\phi$ from $X$ into $Y$ assings to each $x \in X$, an $L$-fuzzy set $\phi(x)$ in $Y$. We can identify with an $L$-fuzzy subset $G_{\phi} \in L^{X \times Y}, \phi(x)(y)=G_{\phi}(x, y)$ for any $(x, y) \in X \times Y$.

Consider an $L$-FMM $\phi$ from $X$ into $Y$. The domain of $\phi$, denoted by $\operatorname{dom}(\phi)$ and the range of $\phi$, denoted by $r n g(\phi)$, for any $x \in X$ and $y \in Y$,

$$
\operatorname{dom}(\phi)(x)=\bigvee_{y \in Y} G_{\phi}(x, y) \text {, and } r n g(\phi)(y)=\bigvee_{x \in X} G_{\phi}(x, y) \text {. }
$$

The inverse mapping of an $L$-FMM $\phi$ from $X$ into $Y$ is an $L$-FMM $\phi^{\leftarrow}$ from $Y$ into $X$ defined by

$$
\begin{aligned}
& \phi^{\leftarrow}: Y \rightarrow L^{X}, \quad \quad \text { where } \phi^{\leftarrow}(y): X \rightarrow L \\
& y \rightarrow \phi^{\leftarrow}(y) \text {, for each } y \in Y, \quad x \rightarrow G_{\phi}(x, y) .
\end{aligned}
$$

One can immediately veirfy that $\operatorname{dom}\left(\phi^{\leftarrow}\right)=r n g(\phi)$ and $\operatorname{dom}(\phi)=r n g\left(\phi^{\leftarrow}\right)$.

Definition 3.2 Let $\phi$ be an $L$-FMM from $X$ into $Y$. Then,

(1) the image of an $L$-fuzzy subset $f$ in $X$ is an $L$-fuzzy subset $\phi^{\Rightarrow}(f)$ in $Y$ is defined by

$$
\phi^{\Rightarrow}(f)(y)=\bigvee_{x \in X}\left[G_{\phi}(x, y) \odot f(x)\right]
$$

(2) the lower inverse of an $L$-fuzzy subset $g$ in $Y$ is an $L$-fuzzy subset $\phi^{\Downarrow}(g)$ in $X$ is defined by

$$
\phi^{\Downarrow}(g)(x)=\bigvee_{y \in Y}\left[G_{\phi}(x, y) \odot g(y)\right] .
$$

(3) the upper inverse of an $L$-fuzzy subset $g$ in $Y$ is an $L$-fuzzy subset $\phi^{\Uparrow}(g)$ in $X$ is defined by

$$
\phi^{\Uparrow}(g)(x)=\bigwedge_{y \in Y}\left[G_{\phi}(x, y) \oplus g(y)\right] .
$$

Definition 3.3 Let $\phi$ be an $L$-FMM from $X$ into $Y$. Then, $\phi$ is called: 
(1) surjective $L$-FMM iff for each $y \in Y, \operatorname{rng}(\phi)(y)=\top$.

(2) a crisp $L$-FMM iff for each $x \in X, G_{\phi}(x, y)=\top$, for any $y \in Y$.

(3) normalized $L$-FMM iff for each $x \in X$, there exists $y_{0} \in Y$ such that $G_{\phi}\left(x, y_{0}\right)=\top$.

Theorem 3.4 Let $\phi$ be an $L$-FMM from $X$ into $Y$. Then,

(1) if $f_{1} \leq f_{2}$, then $\phi \Rightarrow\left(f_{1}\right) \leq \phi \Rightarrow\left(f_{2}\right)$.

(2) if $g_{1} \leq g_{2}$, then $\phi^{\Downarrow}\left(g_{1}\right) \leq \phi^{\Downarrow}\left(g_{2}\right)$ and $\phi^{\Uparrow}\left(g_{1}\right) \leq \phi^{\Uparrow}\left(g_{2}\right)$.

(3) if $\phi$ is normalized, then $\phi^{\Uparrow}(g) \leq \phi^{\Downarrow}(g)$.

(4) if $\phi^{\leftarrow}$ is normalized, then $\left(\phi^{\Rightarrow}(f)\right)^{*} \leq \phi^{\Rightarrow}\left(f^{*}\right)$.

(5) if $\phi$ is normalized, then $\left(\phi^{\Downarrow}(f)\right)^{*} \leq \phi^{\Downarrow}\left(f^{*}\right)$.

(6) $\phi^{\Uparrow}(f)=\left(\phi^{\Downarrow}\left(f^{*}\right)\right)^{*}$ and $\phi^{\Downarrow}(f)=\left(\phi^{\Uparrow}\left(f^{*}\right)\right)^{*}$

Proof (1) and (2) the proof is easy by the definition.

(3) Since $\phi$ is normalized, we can choose $y_{0} \in Y$ such that $G_{\phi}\left(x, y_{0}\right)=\top$. Then,

$$
\begin{aligned}
& \phi^{\Downarrow}(g)(x)=\bigvee_{y \in Y}\left[G_{\phi}(x, y) \odot g(y)\right] \geq G_{\phi}\left(x, y_{0}\right) \odot g\left(y_{0}\right)=\top \odot g\left(y_{0}\right)=g\left(y_{0}\right) . \\
& \phi^{\Uparrow}(g)(x)=\bigwedge_{y \in Y}\left[G_{\phi}^{*}(x, y) \oplus g(y)\right] \leq G_{\phi}^{*}\left(x, y_{0}\right) \oplus g\left(y_{0}\right)=\perp \oplus g\left(y_{0}\right)=g\left(y_{0}\right) .
\end{aligned}
$$

(5) Since $\phi$ is normalized, we can choose $y_{0} \in Y$ such that $G_{\phi}\left(x, y_{0}\right)=\top$. Then,

$$
\begin{aligned}
& \left(\phi^{\Downarrow}(g)\right)^{*}(x)=\left(\bigvee_{y \in Y}\left[G_{\phi}(x, y) \odot g(y)\right]\right)^{*} \leq\left(G_{\phi}\left(x, y_{0}\right) \odot g\left(y_{0}\right)\right)^{*}=\left(\top \odot g\left(y_{0}\right)\right)^{*}=g^{*}\left(y_{0}\right) . \\
& \phi^{\Downarrow}\left(g^{*}\right)(x)=\bigwedge_{y \in Y}\left[G_{\phi}^{*}(x, y) \oplus g(y)\right] \geq G_{\phi}^{*}\left(x, y_{0}\right) \oplus g^{*}\left(y_{0}\right)=\perp \oplus g^{*}\left(y_{0}\right)=g^{*}\left(y_{0}\right) . \\
& \quad(6)\left(\phi^{\Downarrow}\left(f^{*}\right)\right)^{*}(x)=\left(\bigvee_{y \in Y}\left[G_{\phi}(x, y) \odot f(y)\right]\right)^{*} \\
& =\bigwedge_{y \in Y}\left(\left[G_{\phi}(x, y) \odot f^{*}(y)\right]\right)^{*} \\
& =\bigwedge_{y \in Y}\left(\left[G_{\phi}^{*}(x, y) \oplus f(y)\right]\right) \\
& =\phi^{\Uparrow}(f)(x) .
\end{aligned}
$$

Theorem 3.5 Let $(L, \leq, \odot, \oplus, *) \in \mid$ DQUAT $\mid$ and $\phi: X \rightarrow L^{Y}$ be an L-FMM. Then

(1) $\phi \Rightarrow\left(\bigwedge_{i \in \Gamma} f_{i}\right) \leq \bigwedge_{i \in \Gamma} \phi^{\Rightarrow}\left(f_{i}\right)$.

(2) $\phi^{\Downarrow}\left(\bigwedge_{i \in \Gamma} f_{i}\right) \leq \bigwedge_{i \in \Gamma} \phi^{\Downarrow}\left(f_{i}\right)$.

(3) $\phi^{\Uparrow}\left(\bigvee_{i \in \Gamma} f_{i}\right) \geq \bigvee_{i \in \Gamma} \phi^{\Uparrow}\left(f_{i}\right)$.

Proof (1) This can be proved in a similar way as (2). 
(2) $\phi^{\Downarrow}\left(\bigwedge_{i \in \Gamma} f_{i}\right)(x)=\bigvee_{y \in Y}\left[G_{\phi}(x, y) \odot\left(\bigwedge_{i \in \Gamma} f_{i}(y)\right)\right]$

$=\left(\bigvee_{y \in Y} \bigwedge_{i \in \Gamma}\left[G_{\phi}(x, y) \odot f_{i}(y)\right]\right)$

$\leq \bigwedge_{i \in \Gamma}\left(\bigvee_{y \in Y}\left[G_{\phi}(x, y) \odot f_{i}(y)\right]\right)=\bigwedge_{i \in \Gamma} \phi^{\Downarrow}\left(f_{i}\right)$.

(3) $\phi^{\Uparrow}\left(\bigvee_{i \in \Gamma} f_{i}\right)(x)=\bigwedge_{y \in Y}\left[G_{\phi}^{*}(x, y) \oplus\left(\bigvee_{i \in \Gamma} f_{i}(y)\right)\right]$

$=\left(\bigwedge_{y \in Y} \bigvee_{i \in \Gamma}\left[G_{\phi}^{*}(x, y) \oplus f_{i}(y)\right]\right)$

$\geq \bigvee_{i \in \Gamma}\left(\bigwedge_{y \in Y}\left[G_{\phi}^{*}(x, y) \oplus f_{i}(y)\right]\right)=\bigvee_{i \in \Gamma} \phi^{\Uparrow}\left(f_{i}\right)$

Theorem 3.6 Let $\phi: X \rightarrow L^{Y}$ be an $L$-FMM. Then

(1) $\phi \Rightarrow\left(\bigvee_{i \in \Gamma} f_{i}\right)=\bigvee_{i \in \Gamma} \phi^{\Rightarrow}\left(f_{i}\right)$.

(2) $\phi^{\Downarrow}\left(\bigvee_{i \in \Gamma} f_{i}\right)=\bigvee_{i \in \Gamma} \phi^{\Downarrow}\left(f_{i}\right)$.

(3) $\phi^{\Uparrow}\left(\bigwedge_{i \in \Gamma} f_{i}\right)=\bigwedge_{i \in \Gamma} \phi^{\Uparrow}\left(f_{i}\right)$.

(4) $\phi^{\Rightarrow}(\underline{\alpha})=\underline{\alpha}$, if $\phi$ is surjective.

(5) $\phi^{\Downarrow}(\underline{\alpha})=\phi^{\Uparrow}(\underline{\alpha})=\underline{\alpha}$, if $\phi^{\leftarrow}$ is surjective.

$\operatorname{Proof}(5) \phi^{\Downarrow}(\underline{\alpha})=\bigvee_{y \in Y}\left[G_{\phi}(x, y) \odot \underline{\alpha}(y)\right]$

$=\bigvee_{y \in Y}\left[G_{\phi}(x, y) \odot \alpha\right]$

$=\top \odot \alpha=\alpha(y)$,

$\phi^{\Uparrow}(\underline{\alpha})=\bigwedge_{y \in Y}\left[G_{\phi}^{*}(x, y) \oplus \underline{\alpha}(y)\right]$

$=\bigwedge_{y \in Y}\left[G_{\phi}^{*}(x, y) \oplus \alpha\right]$

$=\perp \oplus \alpha=\alpha(y)$.

Definition 3.7 Consider the family $\left\{\phi_{i}\right\}_{i \in \Gamma}$ of $L$-FMM's from $X$ into $Y$, we define the union and the intersection of the family pointwise as follows:

$$
\begin{aligned}
& \bigcup_{i \in \Gamma} \phi_{i}: X \rightarrow Y \text { is defined by }\left(\bigcup_{i \in \Gamma} \phi_{i}\right)(x)=\bigvee_{i \in \Gamma} \phi_{i}(x), \\
& \bigcap_{i \in \Gamma} \phi_{i}: X \rightarrow Y \text { is defined by }\left(\bigcap_{i \in \Gamma} \phi_{i}\right)(x)=\bigwedge_{i \in \Gamma} \phi_{i}(x) .
\end{aligned}
$$

Theorem 3.8 Let $(L, \leq, \odot, \oplus, *) \in \mid$ DQUAT $\mid$ and $\phi_{i}: X \rightarrow L^{Y}$ be an $L$-FMM. Then,

(1) $\left(\bigcap_{i \in \Gamma} \phi_{i}\right) \Rightarrow(f) \leq \bigwedge_{i \in \Gamma}\left(\phi_{i}\right) \Rightarrow(f)$.

(2) $\left(\bigcap_{i \in \Gamma} \phi_{i}\right)_{L}^{\Downarrow}(f) \leq \bigwedge_{i \in \Gamma}\left(\phi_{i}\right)^{\Downarrow}(f)$.

(3) $\left(\bigcap_{i \in \Gamma} \phi_{i}\right)_{L}^{\Uparrow}(f) \geq \bigvee_{i \in \Gamma}\left(\phi_{i}\right)^{\Uparrow}(f)$.

$\operatorname{Proof}(2)\left(\bigcap_{i \in \Gamma} \phi_{i}\right)_{L}^{\Downarrow}(f)(x)=\bigvee_{y \in Y}\left(G_{\left(\bigcap_{i \in \Gamma} \phi_{i}\right)}(x, y) \odot f(y)\right)$

$=\bigvee_{y \in Y}\left(\bigwedge_{i \in \Gamma} \phi_{i}(x)(y) \odot f(y)\right)$

$\leq \bigwedge_{i \in \Gamma}\left(\bigvee_{y \in Y} G_{\phi_{i}}(x, y) \odot f(y)\right)=\bigwedge_{i \in \Gamma}\left(\phi_{i}\right)^{\Downarrow}(f)(x)$.

(3) $\left(\bigcap_{i \in \Gamma} \phi_{i}\right)^{\Uparrow}(f)(x)=\bigwedge_{y \in Y}\left(G_{\left(\bigcap_{i \in \Gamma}^{*} \phi_{i}\right)}(x, y) \oplus f(y)\right)$

$=\bigwedge_{y \in Y}\left(\bigvee_{i \in \Gamma}\left(\phi_{i}(x)(y)\right)^{*} \oplus f(y)\right)$

$\geq \bigvee_{i \in \Gamma}\left(\bigwedge_{y \in Y} G_{\phi_{i}}^{*}(x, y) \oplus f(y)\right)=\bigvee_{i \in \Gamma}\left(\phi_{i}\right)^{\Uparrow}(f)(x)$. 
Theorem 3.9 Let $\phi_{i}: X \rightarrow L^{Y}$ be an $L$-FMM. Then,

(1) $\left(\bigcup_{i \in \Gamma} \phi_{i}\right) \Rightarrow(f)=\bigvee_{i \in \Gamma}\left(\phi_{i}\right) \Rightarrow(f)$.

(2) $\left(\bigcup_{i \in \Gamma} \phi_{i}\right)^{\Downarrow}(f)=\bigvee_{i \in \Gamma}\left(\phi_{i}\right)^{\Downarrow}(f)$.

(3) $\left(\bigcup_{i \in \Gamma} \phi_{i}\right)^{\Uparrow}(f) \leq \bigvee_{i \in \Gamma}\left(\phi_{i}\right)^{\Uparrow}(f)$.

(4) $\left(\bigcup_{i \in \Gamma} \phi_{i}\right)^{\Uparrow}(f)=\bigwedge_{i \in \Gamma}\left(\phi_{i}\right)^{\Uparrow}(f)$.

(5) $\left(\bigcap_{i \in \Gamma} \phi_{i}\right)^{\Uparrow}(f) \neq \bigwedge_{i \in \Gamma}\left(\phi_{i}\right)^{\Uparrow}(f)$.

(6) If $\phi$ is normalized, then $\left(\phi_{i}\right)^{\Downarrow}\left(\left(\phi_{i}\right) \Rightarrow\right)(f) \geq f$.

(7) If $\phi^{\leftarrow}$ is normalized, then $\phi^{\Rightarrow}\left(\left(\phi_{i}\right)^{\Downarrow}\right)(f) \geq f$.

(8) $\left(\phi_{i}\right) \Rightarrow\left(\left(\phi_{i}\right)^{\Downarrow}\right)(f) \nsupseteq f$.

Proof $(2)\left(\bigcup_{i \in \Gamma} \phi_{i}\right)_{L}^{\Downarrow}(f)(x)=\bigvee_{y \in Y}\left(G_{\left(\bigcup_{i \in \Gamma} \phi_{i}\right)}(x, y) \odot f(y)\right)$

$\left.=\bigvee_{y \in Y}\left(\left(\bigcup_{i \in \Gamma} \phi_{i}\right)(x)\right)(y) \odot f(y)\right)$

$=\bigvee_{y \in Y}\left(\bigvee_{i \in \Gamma}\left(\phi_{i}(x)(y) \odot f(y)\right)\right.$

$=\bigvee_{i \in \Gamma}\left(\bigvee_{y \in Y} G_{\phi_{i}}(x, y) \odot f(y)\right)$

$=\bigvee_{i \in \Gamma}\left(\phi_{i}\right)^{\Downarrow}(f)(x)=\bigvee_{i \in \Gamma}\left(\phi_{i}\right)_{L}^{\Downarrow}(f)(x)$.

(3) $\left(\bigcup_{i \in \Gamma} \phi_{i}\right)_{L}^{\Uparrow}(f)(x)=\bigwedge_{y \in Y}\left(G_{\left(\bigcup_{i \in \Gamma}^{*} \phi_{i}\right)}(x, y) \oplus f(y)\right)$

$=\bigwedge_{y \in Y}\left(\left(\bigvee_{i \in \Gamma} \phi_{i}(x)(y)\right)^{*} \oplus f(y)\right)$

$=\bigwedge_{y \in Y}\left(\bigwedge_{i \in \Gamma}\left(\phi_{i}(x)(y)\right)^{*} \oplus f(y)\right)$

$=\bigwedge_{i \in \Gamma}\left(\bigwedge_{y \in Y} G_{\phi_{i}}^{*}(x, y) \oplus f(y)\right)=\bigwedge_{i \in \Gamma}\left(\phi_{i}\right) \Uparrow(f)(x)$.

(6) Since $\phi$ is normalized, then there exists $y_{0}$ such that $G_{\phi}\left(x, y_{0}\right)=\top$. Let $x \in X$ and $f \in L^{X}$. Then, $\left(\phi_{i}\right)^{\Downarrow}\left(\left(\phi_{i}\right) \Rightarrow(f)\right)(x)=\bigvee_{y \in Y}\left[G_{\phi_{i}}(x, y) \odot\left(\phi_{i}\right) \Rightarrow(f)(y)\right]$ $=\bigvee_{y \in Y}\left[G_{\phi_{i}}(x, y) \odot\left(\bigvee_{z \in X}\left[G_{\phi_{i}}(z, y) \odot f(z)\right]\right)\right]$

$\left.\geq \bigvee_{y \in Y}\left[G_{\phi_{i}}(x, y) \odot\left(\left[G_{\phi_{i}}\left(x, y_{0}\right) \odot f(x)\right]\right)\right]\right)$

$=\bigvee_{y \in Y}\left[G_{\phi_{i}}(x, y) \odot f(x)\right]$

$=\left(\bigvee_{y \in Y} G_{\phi_{i}}(x, y)\right) \odot f(x)=\top \odot f(x)=f(x)$.

Example 3.10 Let $L=[0,1]$ be given and $X=\left\{x_{1}, x_{2}\right\}, Y=\left\{y_{1}, y_{2}, y_{3}\right\}$ and $\phi: X \rightarrow L^{Y}$ be $L$-FMM defined by $G_{\phi}\left(x_{1}, y_{1}\right)=0.3, G_{\phi}\left(x_{1}, y_{2}\right)=0.6$, $G_{\phi}\left(x_{1}, y_{3}\right)=0.0, G_{\phi}\left(x_{2}, y_{1}\right)=0.0, G_{\phi}\left(x_{2}, y_{2}\right)=0.0$ and $G_{\phi}\left(x_{2}, y_{3}\right)=0.4$. Define $f\left(y_{1}\right)=0.3, f\left(y_{2}\right)=0.0$ and $f\left(y_{3}\right)=0.7$. Let $x \odot y=0 \vee(x+y-1)$ and $x \oplus y=1 \wedge(x+y)$. Then $\phi^{\Downarrow}\left(x_{1}\right)=\left[G_{\phi}\left(x_{1}, y_{1}\right) \odot f\left(y_{1}\right)\right] \vee\left[G_{\phi}\left(x_{1}, y_{2}\right) \odot\right.$ $\left.f\left(y_{2}\right)\right] \vee\left[G_{\phi}\left(x_{1}, y_{3}\right) \odot f\left(y_{3}\right)\right]$

$=[0.3 \odot 0.3] \vee[0.6 \odot 0.0] \vee[0.0 \odot 0.7]=0.0, \phi^{\Downarrow}\left(x_{2}\right)=\left[G_{\phi}\left(x_{2}, y_{1}\right) \odot f\left(y_{1}\right)\right] \vee$ $\left[G_{\phi}\left(x_{2}, y_{2}\right) \odot f\left(y_{2}\right)\right] \vee\left[G_{\phi}\left(x_{3}, y_{3}\right) \odot f\left(y_{3}\right)\right]$

$=[0.0 \odot 0.3] \vee[0.0 \odot 0.0] \vee[0.4 \odot 0.7]=0.1, \phi^{\Rightarrow} \phi^{\Downarrow}(f)\left(y_{1}\right)=\left[G_{\phi}\left(x_{1}, y_{1}\right) \odot\right.$ $\left.\phi^{\Downarrow}\left(f\left(x_{1}\right)\right)\right] \vee\left[G_{\phi}\left(x_{2}, y_{1}\right) \odot \phi^{\Downarrow}\left(f\left(x_{2}\right)\right)\right]$

$=[0.3 \odot 0.0] \vee[0.0 \odot 0.1]=0.0 \phi^{\Rightarrow} \phi^{\Downarrow}(f)\left(y_{2}\right)=\left[G_{\phi}\left(x_{1}, y_{2}\right) \odot \phi^{\Downarrow}\left(f\left(x_{1}\right)\right)\right] \vee$ $\left[G_{\phi}\left(x_{2}, y_{2}\right) \odot \phi^{\Downarrow}\left(f\left(x_{2}\right)\right)\right]$

$=[0.6 \odot 0.0] \vee[0.0 \odot 0.1]=0.0 \phi^{\Rightarrow} \phi^{\Downarrow}(f)\left(y_{3}\right)=\left[G_{\phi}\left(x_{1}, y_{3}\right) \odot \phi^{\Downarrow}\left(f\left(x_{1}\right)\right)\right] \vee$ $\left[G_{\phi}\left(x_{2}, y_{3}\right) \odot \phi^{\Downarrow}\left(f\left(x_{2}\right)\right)\right]$

$=[0.0 \odot 0.0] \vee[0.4 \odot 0.1]=0.0$ Thus, $\phi^{\Rightarrow} \phi^{\Downarrow}(f) \not f$.

Theorem 3.11 Let $\phi: X \rightarrow L^{Y}$ be a crisp $L$-FMM. Then 
(1) $\phi \Rightarrow\left(\phi^{\Uparrow}(f)\right) \leq f$.

(2) $\phi^{\Uparrow}(\phi \Rightarrow(f)) \geq f$.

(3) If $\phi$ is normalized, then $\phi^{\Downarrow}\left(\phi^{\Rightarrow}(\underline{\alpha})\right)=\underline{\alpha}$.

(4) If $\phi^{\Downarrow}$ is normalized, then $\phi^{\Rightarrow}\left(\phi^{\Downarrow}(\underline{\alpha})\right)=\underline{\alpha}$.

Proof $(1) \phi^{\Rightarrow}\left(\phi^{\Uparrow}(f)\right)=\bigvee_{x \in X}\left[G_{\phi}(x, y) \odot \phi^{\Uparrow}(f)(x)\right]$

$=\bigvee_{x \in X}\left[G_{\phi}(x, y) \odot \bigwedge_{s \in Y}\left[G_{\phi}^{*}(x, s) \oplus f(s)\right]\right]$

$\leq \bigvee_{x \in X}\left[G_{\phi}(x, y) \odot\left[G_{\phi}^{*}(x, y) \oplus f(y)\right]\right]$

$\leq \bigvee_{x \in X}\left[G_{\phi}(x, y) \wedge\left[G_{\phi}^{*}(x, y) \oplus f(y)\right]\right]$

$=f(y) \cdot$ Thus,$\phi^{\Rightarrow}\left(\phi^{\Uparrow}(f)\right) \leq f$.

(2) $\phi^{\Uparrow}\left(\phi \Rightarrow(f)=\bigwedge_{y \in Y}\left[G_{\phi}^{*}(x, y) \oplus \phi^{\Rightarrow}(f)(y)\right]\right.$

$=\bigwedge_{y \in Y}\left[G_{\phi}^{*}(x, y) \oplus \bigvee_{z \in X}\left[G_{\phi}(z, y) \odot f(z)\right]\right]$

$\geq \bigwedge_{y \in Y}\left[G_{\phi}^{*}(x, y) \oplus\left[G_{\phi}(x, y) \odot f(x)\right]\right]$

$\geq \bigwedge_{y \in Y}\left[G_{\phi}^{*}(x, y) \vee\left[G_{\phi}(x, y) \odot f(x)\right]\right]$

$=f(x) \cdot T h u s \phi^{\Uparrow}\left(\phi^{\Rightarrow}(f)\right) \geq f$.

(3) Since $\phi$ is normalized, then there exists $y_{0}$ such that $G_{\phi}\left(x, y_{0}\right)=\top$. Let $x \in X$ and $f \in L^{X}$. Then we have

$$
\begin{aligned}
& \phi^{\Downarrow}\left(\phi^{\Rightarrow}(\underline{\alpha})\right)(x)=\bigvee_{y \in Y}\left[G_{\phi}(x, y) \odot \phi^{\Rightarrow}(\underline{\alpha})(y)\right] \\
= & \bigvee_{y \in Y}\left[G_{\phi}(x, y) \odot\left(\bigvee_{z \in X}\left[G_{\phi}(z, y) \odot \underline{\alpha}(z)\right]\right)\right] \\
= & \bigvee_{y \in Y}\left[G_{\phi}(x, y) \odot\left(\left(\bigvee_{z \in X} G_{\phi}(z, y)\right) \odot \alpha\right)\right] \\
= & {\left[\left(\bigvee_{y \in Y-\left\{y_{0}\right\}}\left(G_{\phi}(x, y) \odot \bigvee_{z \in X} G_{\phi}(z, y)\right) \bigvee\left(G_{\phi}\left(x, y_{0}\right) \odot \bigvee_{z \in X} G_{\phi}\left(z, y_{0}\right)\right)\right)\right] \odot \alpha } \\
= & \left(\bigvee_{y \in Y-\left\{y_{0}\right\}}\left(G_{\phi}(x, y) \odot \bigvee_{z \in X} G_{\phi}(z, y)\right) \vee \top\right) \odot \alpha \\
= & \top \odot \underline{\alpha}(x)=\underline{\alpha}(x) .
\end{aligned}
$$

Definition 3.12 Let $\phi: X \rightarrow L^{Y}$ and $\psi: Y \rightarrow L^{Z}$ be two $L$-FMM's. Then the composition $\psi \circ \phi$ is defined by

$$
((\psi \circ \phi) \underset{L}{\Rightarrow}(x))(z)=\bigvee_{y \in Y}\left[G_{\phi}(x, y) \odot G_{\psi}(y, z)\right]
$$

Theorem 3.13 Let $\phi: X \rightarrow L^{Y}$ and $\psi: Y \rightarrow L^{Z}$ be two $L$-FMM's. Then we have the following

(1) $((\psi \circ \phi) \vec{L})=\phi \Rightarrow(\psi \vec{L})$.

(2) $\left((\psi \circ \phi)_{L}^{\Uparrow}\right)=\phi^{\Uparrow}\left(\psi_{L}^{\Uparrow}\right)$.

(3) $\left((\psi \circ \phi)_{L}^{\Downarrow}\right)=\phi^{\Downarrow}\left(\psi_{L}^{\Downarrow}\right)$.

Proof (2) Let $x \in X$ and $f \in L^{X}$. Then we have

$$
\begin{aligned}
& \left((\psi \circ \phi)_{L}^{\Uparrow}\right)=\bigwedge_{z \in Z}\left[\left(G_{\psi \circ \phi}(x, z)\right)^{*} \oplus f(z)\right] \\
= & \bigwedge_{z \in Z}\left((\psi \circ \phi) \overrightarrow{\vec{L}}_{L}(x)(z)\right)^{*} \oplus f(z) \\
= & \bigwedge_{z \in Z}\left(\left(\bigvee_{y \in Y}\left[G_{\phi}(x, y) \odot G_{\psi}(y, z)\right]\right)^{*} \oplus f(z)\right) \\
= & \bigwedge_{z \in Z} \bigwedge_{y \in Y}\left(\left[\left(G_{\phi}(x, y)\right)^{*} \oplus\left(G_{\psi}(y, z)\right)^{*}\right] \oplus f(z)\right)
\end{aligned}
$$




$$
\begin{aligned}
& =\bigwedge_{y \in Y}\left(G_{\phi}(x, y)\right)^{*} \oplus\left[\bigwedge_{z \in Z}\left(G_{\psi}(y, z)\right)^{*} \oplus f(z)\right] \\
& =\bigwedge_{y \in Y}\left(G_{\phi}(x, y)\right)^{*} \oplus \psi_{L}^{\Uparrow}(f)(y) \\
& =\phi^{\Uparrow}\left(\psi_{L}^{\Uparrow}(f)(x) . \text { Thus },\left((\psi \circ \phi)_{L}^{\Uparrow}\right)=\phi^{\Uparrow}\left(\psi_{L}^{\Uparrow}\right) .\right.
\end{aligned}
$$

(3) From Theorem 3.4 $(6),(\psi \circ \phi)_{L}^{\Downarrow}(f)=\left((\psi \circ \phi)_{L}^{\Uparrow}\left(f^{*}\right)\right)^{*}=\left(\phi^{\Uparrow}\left(\psi_{L}^{\Uparrow}\left(f^{*}\right)\right)\right)^{*}=$ $\left(\phi^{\Uparrow}\left(\psi_{L}^{\Downarrow}(f)\right)^{*}\right)^{*}=\phi^{\Downarrow}\left(\psi_{L}^{\Downarrow}(f)\right.$.

\section{References}

[1] Elena Tsiporkova-Hristoskova, Bernard De Baets and Etienne Kerre, A Detailed study of direct and inverse images under fuzzy multivalued mappings, J. Fuzzy Math,3(1)(1995), 191-208.

[2] Elena Tsiporkova-Hristoskova and Etienne Kerre, Pure and stable fuzzy sets, Proceeding of the international AMSE converence "Systems Analysis, Control, Design", Symposium on "Fuzzy Systems, Neural Networks" Lyon, France, July 4-6( 1994), 2-12.

[3] Elena Tsiporkova-Hristoskova, Bernard De Baets, Etienne Kerre, Fuzzy lower semi-continuity of fuzzy multivalued mappings, Proceeding of the EUFIT'49 second European Congress on Intelligent Techniques and Soft Computing (Aachen, Germany, September 20-23(1994), 1341-1346.

[4] P. Ottoy and E. Kerre, On the fuzzification of multivalued mappings, Proceedings of the workshop on Knowledge-Based systems and models of Logical resoning (Cairo, Egypt, December 26-31,1988.

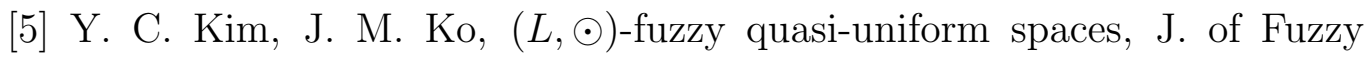
Math.16(1)(2008), 163-184.

[6] S.E.Rodabaugh, Axiomatic foundations for uniform operator quasiuniformities, in: E.P. Klement, S.E.Rodabaugh (Eds.), Topological and Algebraic structures in fuzzy sets, The Handbook of Recent Developments in the Mathematics of Fuzzy Sets, Trends in Logic Vol. 20, Kluwer Academic Publishers, (Boston/Dordrecht/London) (2003),199-234.

\section{Received: October 1, 2014}

